\title{
Isolated Horner syndrome as a rare initial presentation of nasopharyngeal carcinoma: a case report
}

\author{
Tanyatuth Padungkiatsagul' \\ Anuchit Poonyathalang' \\ Panitha Jindahra ${ }^{2}$ \\ Piyaphon Cheecharoen ${ }^{3}$ \\ Kavin Vanikieti' \\ 'Department of Ophthalmology, \\ Faculty of Medicine Ramathibodi \\ Hospital, Mahidol University, Bangkok, \\ Thailand; ' ${ }^{2}$ Department of Medicine, \\ Faculty of Medicine Ramathibodi \\ Hospital, Mahidol University, Bangkok, \\ Thailand; ${ }^{3}$ Department of Radiology, \\ Faculty of Medicine Ramathibodi \\ Hospital, Mahidol University, Bangkok, \\ Thailand
}

This article was published in the following Dove Press journal: International Medical Case Reports Journal

\begin{abstract}
Background: Horner syndrome refers to a set of clinical presentations resulting from disruption of sympathetic innervation to the eye and adnexa. Classically, the clinical triad consists of ipsilateral blepharoptosis, pupillary miosis, and facial anhidrosis. Ocular sympathetic denervation may signify life-threatening causes. Timely investigation and accurate diagnosis are essential in patients with oculosympathetic denervation.
\end{abstract}

Case presentation: A 33-year-old Asian man with a heavy smoking habit presented with a 3 -week history of left ptosis and no other complaints. His visual acuity was 20/20 bilaterally. An ophthalmic examination was significant for mild ptosis of his left eyelid and anisocoria (smaller left pupil), which was greater in the dark. Both pupils reacted to light briskly without an afferent pupillary defect. Anhidrosis was found on the medial side of the left forehead. A $10 \%$ cocaine test was positive. At his first visit, neurologic examination was unremarkable. Comprehensive radiological investigations were scheduled for a left-sided isolated Horner syndrome. Two weeks after his first visit, he experienced a left-sided headache along with ipsilateral Horner syndrome. Neurologic examination revealed hypoesthesia in the left cranial nerve $\mathrm{V}_{1-3}$ territories. Emergent computed tomography angiography was suspected for petrous part of the left internal carotid artery (ICA) dissection. Magnetic resonance imaging demonstrated an enhancing infiltrative lesion with its epicenter at the left sphenoid bone. The lesion encased the left ICA and invaded the left Meckel cave. Rhinoscopy with incisional biopsy revealed squamous cell nasopharyngeal carcinoma.

Conclusion: This case involved an unusual initial presentation of nasopharyngeal carcinoma: isolated Horner syndrome with clinical progression to adjacent structures. Infiltration involving the Meckel cave and ICA at the foramen lacerum can present as postganglionic Horner syndrome associated with trigeminal pain and hypoesthesia. These clinical findings may mimic carotid artery dissection on computed tomography angiography. Detailed magnetic resonance imaging with careful attention to the skull base should be performed.

Keywords: Horner syndrome, nasopharyngeal carcinoma, trigeminal

\section{Background}

Horner syndrome, or oculosympathoparesis, was first described by Johann Friedrich Horner in 1929. It is characterized by a classic constellation of signs comprising ipsilateral blepharoptosis, pupillary miosis, and facial anhidrosis. ${ }^{1}$ The syndrome can result from any disruption of the sympathetic innervation to the eye and ocular adnexa. Differential diagnoses for the pathology responsible for the sympathetic disruption range from benign to life-threatening conditions such as carotid artery dissection and malignancies. ${ }^{2}$ Timely and accurate diagnosis is essential to provide appropriate management.
Correspondence: Kavin Vanikieti Department of Ophthalmology, Faculty of Medicine Ramathibodi Hospital, Mahido University, 270 Rama VI Road, Bangkok 10400, Thailand

Tel +6622011526

Fax +6622012729

Email kavin62@hotmail.com 
A definitive diagnosis of Horner syndrome may require pharmacologic confirmation because of the variable clinical findings of sympathetic denervation of the head and eye. Cocaine - a presynaptic norepinephrine reuptake inhibitor - produces mydriasis, lid retraction, and blanching of the conjunctiva in the normal eye, whereas it produces no response in the pathologic eye. Post-cocaine anisocoria of $\geq 0.8 \mathrm{~mm}$ confirms the presence of oculosympathetic denervation. ${ }^{3}$

The etiologies of Horner syndrome are numerous due to the long and circuitous course of the involved nerves. The three-neuron pathway initiates in the hypothalamus and terminates in the eye. Localization of pathologic site can be inferred by associated clinical findings. Vertigo, ataxia, sensory deficits, dysphagia, or nystagmus suggests a lesion of a central or first-order neuron. Hemifacial anhidrosis, a mass in the anterior neck, hand weakness, or a history of central venous catheterization suggests a lesion of a preganglionic second-order neuron. An ipsilateral headache or neck pain and the absence of anhidrosis (which may present in the medial part of the forehead) suggest damage of a postganglionic third-order neuron.

We herein report a case involving isolated Horner syndrome as an unusual presenting sign of nasopharyngeal carcinoma (NPC). Trigeminal pain and hypoesthesia subsequently developed due to progression of the tumor. Diagnostic procedures and localization of the lesion are discussed in this report.

\section{Case presentation}

A 33-year-old Thai man with a heavy smoking habit presented with a 3-week history of ptosis in his left eye without any other complaints. His visual acuity was $20 / 20$ bilaterally. His intraocular pressure and ocular motility were unremarkable. Ophthalmic examination was significant for mild ptosis of the left eyelid with anisocoria (smaller pupil in the left eye) as shown in Figure 1. The difference in the pupillary diameter between the right and left sides was greater in dark than in bright conditions. In bright light, the right and left pupils were 3 and $2 \mathrm{~mm}$, respectively (Figure 2A). Under dim light, however, the right pupil dilated to $4 \mathrm{~mm}$, whereas the left pupil was almost unchanged $(2.5 \mathrm{~mm})$, as shown in Figure 2B. This finding was the result of iris dilator impairment in the left eye. Both pupils reacted to light briskly without an afferent pupillary defect. Anhidrosis was noted on the medial side of the left forehead. A Horner syndrome confirmatory test (the $10 \%$ cocaine test) was positive; the difference in pupillary diameter increased to $2.5 \mathrm{~mm}(6.0 \mathrm{~mm}$ on the right and $3.5 \mathrm{~mm}$ on the left) after the instillation of

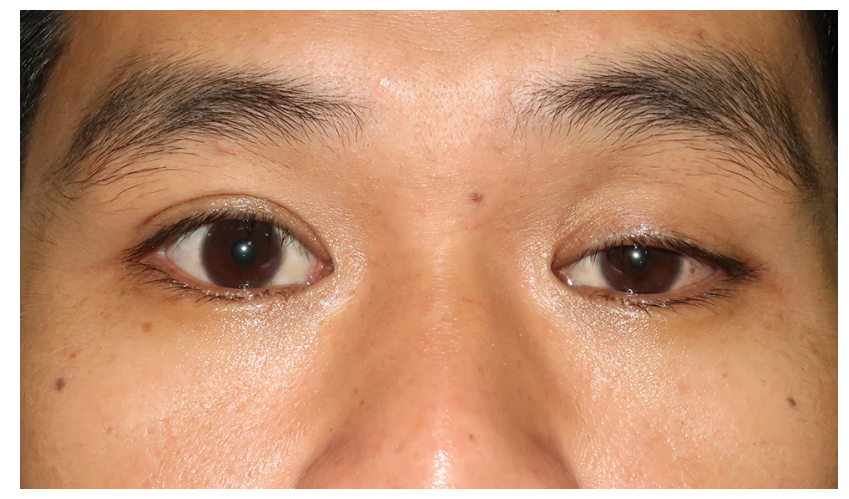

Figure I Primary position shows mild ptosis of the left eyelid with orthotropic ocular alignment.

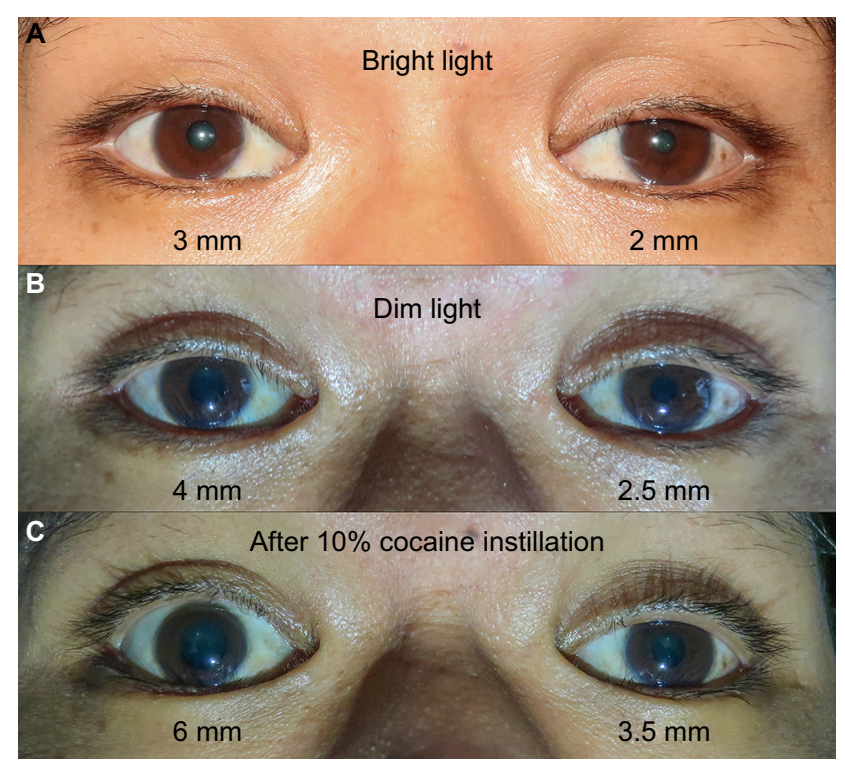

Figure 2 Pupillary examination shows anisocoria.

Notes: (A) In bright light, the right and left pupil diameters are 3 and $2 \mathrm{~mm}$, respectively. (B) The degree of anisocoria is greater in a dark environment, in which the right pupil dilates to $4.0 \mathrm{~mm}$ compared with $2.5 \mathrm{~mm}$ of the left pupil. (C) After $10 \%$ cocaine instillation to both eyes, the difference between the pupils' diameters is $2.5 \mathrm{~mm}$. The positive 10\% cocaine test confirms diagnosis of left Horner syndrome.

$10 \%$ cocaine bilaterally (Figure $2 \mathrm{C}$ ). This was accompanied by right upper eyelid retraction (Figure 2C). At his first visit, physical examination (including a neurologic examination) was unremarkable. Thus, a provisional diagnosis of left-sided painless isolated Horner syndrome was made. Comprehensive radiological investigations including magnetic resonance imaging (MRI) and magnetic resonance angiography of his neck and brain combined with computed tomography of the chest were scheduled. Two weeks after his first visit, he developed a left-sided headache along with ipsilateral Horner syndrome. Neurologic examination revealed hypoesthesia in the left cranial nerve $\mathrm{V}_{1-3}$ territories. Emergent computerized tomography angiography (CTA) of the neck, including the 
skull base, revealed long segmental luminal narrowing of the petrous part of the left internal carotid artery (ICA), and left ICA dissection was suspected (Figure 3A and B). Further MRI and magnetic resonance angiography demonstrated an enhancing infiltrative lesion epicenter at the central skull base, specifically the left sphenoid bone. The lesion encased the left ICA with extensions to the left Meckel cave, left sphenoid wing, and lateral wall of the nasopharynx (Figure 4A and B). Rhinoscopy with incisional biopsy was performed, and pathologic examination revealed undifferentiated, nonkeratinizing, and squamous cell NPC. According to the eighth edition of the American Joint Committee on Cancer staging manual, ${ }^{4}$ his NPC staging was $\mathrm{T}_{4} \mathrm{~N}_{2} \mathrm{M}_{0}$. Tumor was locally advanced with intracranial extension; regional lymph nodes were bilaterally metastasized above the cricoid cartilage level; and investigations revealed no distant metastasis.

\section{Discussion and conclusion}

Horner syndrome is characterized by ipsilateral blepharoptosis, pupillary miosis with anisocoria that is greater in the dark, and facial anhidrosis on the affected side. Establishing the diagnosis and localizing the disruption of the oculosympathetic pathway are essential. Disruption of the three-neuron pathway can be divided into three types: central, preganglionic, and postganglionic. ${ }^{2,5,6}$

The first-order neuron originates from the hypothalamus and travels from the brain stem through the ciliospinal center of Budge-Waller, located at the spinal cord levels C8-T2. ${ }^{6}$ Central causes of Horner syndrome are commonly accompanied by neurologic signs and symptoms. Ataxia, sensory loss, vertigo, vestibular nystagmus, and dysphagia are usually present in patients with Horner syndrome caused by a brain stem lesion. Brain stem infarcts are the most common cause
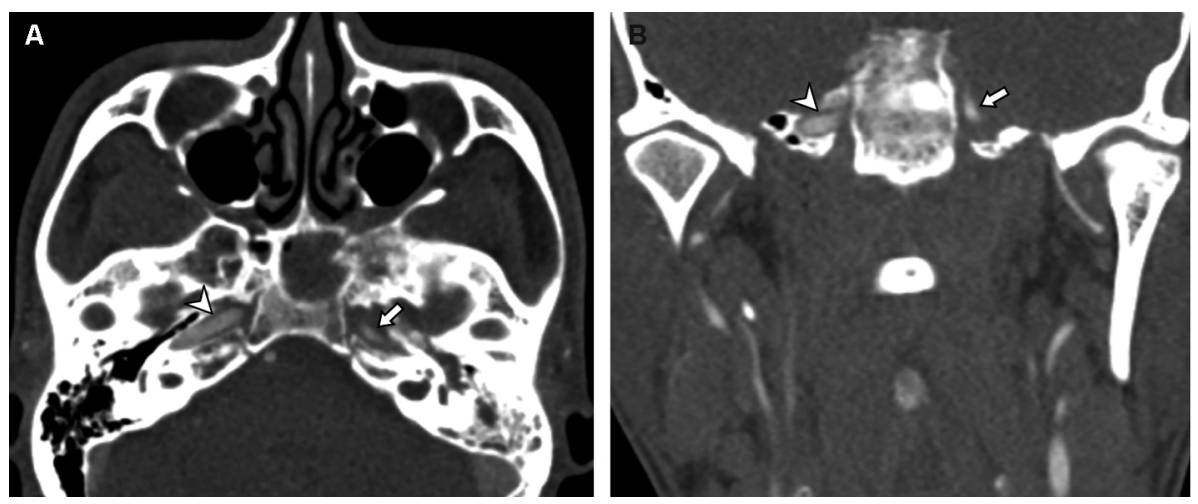

Figure 3 Emergent computed tomography angiography of the neck, including the base of the skull.

Notes: Contrast-enhanced (A) axial and (B) coronal studies show long segmental luminal narrowing of the petrous part of the left ICA (arrows) compared with the normal luminal size of the right ICA (arrowheads). Left ICA dissection is suspected.

Abbreviation: ICA, internal carotid artery.
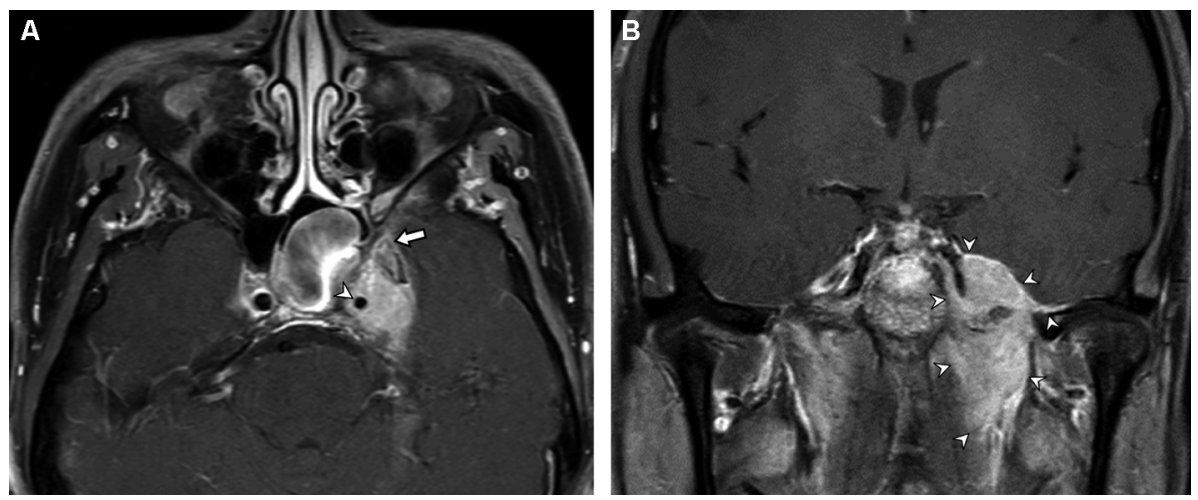

Figure 4 Magnetic resonance imaging of the brain, orbit, and neck.

Notes: (A) Axial TI-weighted imaging with gadolinium injection shows a large infiltrative lesion with strong enhancement encasing the left ICA (arrowhead) and causing segmental luminal narrowing with invasion to the left Meckel cave and left sphenoid wing (arrow). (B) Coronal TI-weighted imaging with gadolinium injection shows that the epicenter of the enhancing infiltrative lesion involves the central skull, and the lesion extends to the medial left middle cranial fossa and left Meckel cave (arrowheads) with extensions to the pterygopalatine fossa and lateral wall of the nasopharynx (not shown).

Abbreviation: ICA, internal carotid artery. 
of central Horner syndrome. ${ }^{7}$ Our patient had no associated brain stem neurologic signs or symptoms, except ipsilateral loss of facial sensation in the cranial nerve $\mathrm{V}_{1-3}$ territories. Therefore, a central lesion was considered unlikely.

After the preganglionic neuron exits the ciliospinal center of Budge-Waller, it travels down through the pulmonary apex and turns upward within the carotid sheath to superior cervical ganglion. Horner syndrome accompanied by ipsilateral shoulder pain should raise the suspicion for neoplastic involvement of the pulmonary apex, pleura, and brachial plexus. Various neck and cardiothoracic surgical procedures that can reportedly cause preganglionic Horner syndrome include coronary artery bypass surgery, carotid endarterectomy, intracostal chest tube insertion, and internal jugular catheterization. ${ }^{2}$ Our patient had neither a history of prior surgical procedures nor clinical manifestations of thoracic abnormalities, including chest pain or hemoptysis. Moreover, anhidrosis was reported only in the medial part of his forehead; other areas of his face were normal. Preganglionic Horner syndrome was, therefore, unlikely in this patient.

The postganglionic third-order neuron starts from the superior cervical ganglion as a sympathetic plexus traveling along the ICA. The fibers responsible for sweating of the face (sudomotor fibers) exit the superior cervical ganglion with the external carotid artery. The remaining sympathetic plexus joins the ICA and enters the skull base through the carotid canal and foramen lacerum and then travels up into the cavernous sinus. At the posterior part of the cavernous sinus, the sympathetic fibers briefly travel with the abducens nerve before joining the ophthalmic division of the trigeminal nerve and entering the orbit. A lesion at the posterior part of the cavernous sinus can produce postganglionic Horner syndrome with abducens paresis, ${ }^{8}$ the so-called Parkinson sign. Most of the sympathetic fibers continue from the nasociliary nerve to the long ciliary nerve and innervate various ocular and orbital structures. Anhidrosis in patients with postganglionic Horner syndrome is usually absent or may be found in the medial part of the forehead because innervation of this area by sudomotor fibers is derived from the postganglionic neuron, in contrast to anhidrosis of the entire ipsilateral face in patients with central or preganglionic Horner syndrome. Differential diagnoses of painful postganglionic Horner syndrome always include life-threatening carotid artery dissection, cluster headache, a cavernous sinus lesion, and Raeder paratrigeminal neuralgia. ${ }^{2,6}$

Our patient initially presented with painless, left-sided, isolated Horner syndrome, comprising miosis with anisocoria that was greater in the dark (Figure 2A and B), mild ipsilateral ptosis (Figure 1), and anhidrosis in the medial part of the ipsilateral forehead. The patient's blepharoptosis was subtle and could be explained by paralysis of Müller's muscle, which is responsible for only $2 \mathrm{~mm}$ of eyelid elevation., ${ }^{2,7}$ The diagnosis of Horner syndrome (oculosympathetic denervation) is usually based on clinical characteristics. Nonetheless, the cocaine test is still a reliable gold standard test with which to confirm the diagnosis. The $10 \%$ cocaine test confirmed the diagnosis of left-sided Horner syndrome in our patient (Figure 2C).

After confirmation with the topical cocaine test, the lesion can be localized with the hydroxyamphetamine test. This test can be used to distinguish a postganglionic lesion from preganglionic and central lesions by hydroxyamphetamine's action of releasing norepinephrine from the presynaptic terminal. Only an intact postganglionic neuron can produce mydriasis. The hydroxyamphetamine test is unavailable in our center. This test is now rarely performed because of its high false-positive and false-negative rates. ${ }^{9}$ Therefore, the clinician must localize the lesion causing Horner syndrome based upon clues from the patient's history and examination. ${ }^{2,7}$ At our patient's first visit, the physical examination, including a neurologic examination, was unremarkable. A provisional diagnosis of painless, left-sided, and isolated Horner syndrome was made. Based solely on the patient's clinical signs, it was difficult to clearly distinguish between a preganglionic and postganglionic lesion. Retrospectively, only the patient's heavy smoking history raised suspicion for NPC. Two weeks after his first visit, he developed a dull, leftsided headache and ipsilateral hypoesthesia in the left cranial nerve $\mathrm{V}_{1-3}$ territories, leading to localization of a third-order neuron lesion (specifically at the left skull base). However, life-threatening carotid artery dissection must be ruled out in patients with postganglionic Horner syndrome with an ipsilateral headache or facial pain. Our patient underwent emergent CTA of the neck, including the skull base. CTA revealed long segmental luminal narrowing of the petrous part of the left ICA, and left ICA dissection was suspected (Figure 3). Further MRI of the brain and neck demonstrated a large enhancing infiltrative lesion encasing the left ICA, causing segmental luminal narrowing with invasion to the central skull, and extending to the left Meckel cave, left sphenoid wing, pterygopalatine fossa, and the lateral wall of nasopharynx (Figure 4). NPC along with other head and neck malignancies were suspected. Rhinoscopy with incisional biopsy was performed, and pathologic examination showed undifferentiated, non-keratinizing, squamous cell NPC.

The age at onset of NPC is usually $40-60$ years. ${ }^{10}$ Men are affected twice as often as women. ${ }^{10}$ The lack of specific 
initial symptoms and slow growth are responsible for the delay in diagnosis of NPC. Otorhinologic symptoms and signs are present in about half of the patients. ${ }^{11-13}$ The tumor typically arises from the roof or lateral wall of the nasopharynx. NPC can disrupt the oculosympathetic pathway at either the preganglionic level through the cervical lymph node and sympathetic plexus involvement ${ }^{12}$ or the postganglionic level. The third-order neuron can be damaged by infiltration of NPC in the skull base and cavernous sinus area, including involvement of various branches of the trigeminal nerve. ${ }^{13-16}$ NPC rarely presents initially as isolated Horner syndrome, as shown in the present case. ${ }^{15,16}$ NPC should also be strongly suspected in any patient who presents with unilateral facial pain associated with ipsilateral trigeminal neuropathy and postganglionic Horner syndrome. ${ }^{12}$ Detailed MRI with careful attention to the skull base should be performed in such cases.

\section{Ethics approval and consent}

This study was approved by the Institutional Review Board of Faculty of Medicine Ramathibodi Hospital. Written informed consent was obtained from the patient for publication of this case report and any accompanying images.

\section{Data sharing statement}

Data for this case report were collected by chart review of the patient's electronic medical record, which is not publicly available because of privacy considerations.

\section{Acknowledgments}

We thank Angela Morben, DVM, ELS, from Edanz Group (www.edanzediting.com/ac), for editing a draft of this manuscript and Nilnetre Mahathanaruk, MD for reviewing the TNM staging of nasopharyngeal carcinoma. This study was conducted at the Department of Ophthalmology, Faculty of Medicine Ramathibodi Hospital, Mahidol University.

\section{Author contributions}

All authors made substantial contributions to the conception and design of the study. TP, PC, and KV contributed to the acquisition of data. All authors contributed to data analysis and interpretation of data. All authors took part in drafting the work and TP and KV revised it critically. All authors gave final approval for submission; and are accountable for all aspects of the work in ensuring that questions related to the accuracy or integrity of any part of the work are appropriately investigated and resolved.

\section{Disclosure}

The authors report no conflicts of interest in this work.

\section{References}

1. Fulton JF. Horner and the syndrome of paralysis of the cervical sympathetic. Arch Surg. 1929;18(4):2025-2039.

2. Kawasaki A. Disorders of pupillary function, accommodation, and lacrimation. In: Miller NR, Newman NJ, editors. Walsh \& Hoyt's Clinical Neuro-Ophthalmology. 6th ed. Philadelphia: Lippincott Williams \& Wilkins; 2005:749-758.

3. Kardon RH, Denison CE, Brown CK, Thompson HS. Critical evaluation of the cocaine test in the diagnosis of Horner's syndrome. Arch Ophthalmol. 1990;108(3):384-387.

4. Amin MB, Greene FL, Edge SB, et al. The Eighth Edition AJCC Cancer Staging Manual: continuing to build a bridge from a population-based to a more "personalized" approach to cancer staging. CA Cancer JClin. 2017;67(2):93-99.

5. Liu GT, Volpe NJ, Galetta SL. Neuro-Ophthalmology: Diagnosis and Management. 2nd ed. Philadelphia: Saunders Elsevier; 2010:428-441.

6. Walton KA, Buono LM. Horner syndrome. Curr Opin Ophthalmol. 2003;14(6):357-363.

7. Lee AG, Brazis PW. Clinical Pathway in Neuro-Ophthalmology. New York: Thieme Medical Publisher Inc.; 2003:445-456.

8. Preechawat P, Poonyathalang A, Boontantrapiwat S, Dhanachai M, Luxameechanporn T. Horner syndrome and abducens nerve paresis due to a paranasal sinus squamous cell carcinoma involving cavernous sinus. Neuroophthalmology. 2009;33(4):185-187.

9. Moster ML, Galiani D, Garfinkle W. False negative hydroxyamphetamine test in Horner syndrome caused by acute internal carotid artery dissection. J Neuroophthalmol. 2003;23(1):22-23.

10. Smith JL, Wheliss JA. Ocular manifestations of nasopharyngeal tumors. Trans Am Acad Ophthalmol Otolaryngol. 1962;66:659-664.

11. Neel HB III. A prospective evaluation of patients with nasopharyngeal carcinoma: an overview. J Otolaryngol. 1986;15(3):137-144.

12. Frishberg B. Miscellaneous tumors of neuro-ophthalmologic interest. In: Miller NR, Newman NJ, editors. Walsh \& Hoyt's Clinical NeuroOphthalmology. 6th ed. Philadelphia: Lippincott Williams \& Wilkins; 2005:1684-1686.

13. Neel HB III, Taylor WF. Clinical presentation and diagnosis of nasopharyngeal carcinoma: current status. In: Prasad U, editor. Nasopharyngeal Carcinoma: Current Concepts. Kuala Lumpur: University of Malaya Press; 1983:1-10.

14. Degirmenci E, Erdogan C, Aras D, Oğuzhanoğlu A. Nasopharyngeal carcinoma presenting with Horner syndrome and carotid-sinus syncope. Neurologist. 2012;18(4):208-210.

15. Murchison AP, Rosen MR, Bilyk JR. Horner syndrome as a presenting sign of nasopharyngeal carcinoma. Ophthalmic Plast Reconstr Surg. 2009;25(5):401-402.

16. Jiménez-Caballero PE, Marsal-Alonso C, Alvarez-Tejerina A. Horner syndrome as the first symptom of nasopharyngeal cancer. Two case reports. Rev Neurol. 2005;40(9):541-543. 
The International Medical Case Reports Journal is an international, peer-reviewed open-access journal publishing original case reports from all medical specialties. Previously unpublished medical posters are also accepted relating to any area of clinical or preclinical science. Submissions should not normally exceed 2,000 words or
4 published pages including figures, diagrams and references. The manuscript management system is completely online and includes a very quick and fair peer-review system, which is all easy to use. Visit http://www.dovepress.com/testimonials.php to read real quotes from published authors.

Submit your manuscript here: https://www.dovepress.com/international-medical-case-reports-journal-journal 